Meta

Journal des tradlucteurs

Translators' Journal

\title{
Féminisme et féminisation des titres : un faux parallèle
}

\section{Donald Geenen}

Volume 23, numéro 2, juin 1978

URI : https://id.erudit.org/iderudit/002835ar

DOI : https://doi.org/10.7202/002835ar

Aller au sommaire du numéro

Éditeur(s)

Les Presses de l'Université de Montréal

\section{ISSN}

0026-0452 (imprimé)

1492-1421 (numérique)

Découvrir la revue

Citer cet article

Geenen, D. (1978). Féminisme et féminisation des titres : un faux parallèle. Meta, 23(2), 163-164. https://doi.org/10.7202/002835ar d'utilisation que vous pouvez consulter en ligne.

https://apropos.erudit.org/fr/usagers/politique-dutilisation/ 


\section{FÉMINISME ET FÉMINISATION DES TITRES: UN FAUX PARALLÈLE}

La présente communication a pour objet d'apporter certains éléments au débat sur la féminisation des titres de fonctions et postes usites dans l'entreprise et dans la vie professionnelle en général.

Rappelons, pour commencer, que les catégories grammaticales appelées « masculin » et « féminin» ne sont que des cas, des flexions, et n'ont aucune valeur « sexuelle », encore moins « sexiste». C'est ainsi qu'une même entité, le «soleil » est masculin en français et féminin en allemand. Ce n'est que depuis la systématisation des corpus linguistiques, et singulièrement depuis l'établissement de la grammaire, qu'à coté des autres flexions (les déclinaisons de nominatif, accusatif, datif et génitif, par exemple, qui existent encore en allemand), on a désigné, sans doute par analogie (consonance des mots, forme et importance des objets designés, etc), les « genres » de substantifs par les termes de « masculin », « féminin » et «neutre ». Que les premiers locuteurs des langues mondiales aient procédé à ce genre d'analogies, en personnifiant par exemple les éléments de la nature sous forme de divinités masculines ou féminines, n'est pas une raison pour que nous, " esprits éclairés » de ce $\mathrm{xx}^{\mathrm{e}}$ siècle finissant, continuions à verser dans la même mythologie. Le fait, par ailleurs, que le français compte un grand nombre de substantifs possédant une flexion « féminine » permettant de désigner d'emblée les êtres (humains ou animaux) de sexe féminin sans devoir recourir à des rallonges explétives du genre he-cat - she-cat ne doit pas faire oublier qu'il présente une faiblesse par rapport à certaines autres langues vivantes (sans compter le latin, dont il est dérivé), qui possèdent encore un genre « neutre ». Or, pourquoi notre catégorie grammaticale « masculin » n'occuperaitelle pas, dans certains cas, la place d'une nouvelle catégorie "masculin-féminin », d'un « neutre » réinventé, et singulièrement lorsqu'il s'agit de titres, de désignations de postes pouvant être occupés (autre innovation fort heureuse) tant par des hommes que par des femmes? Rappelons, à cet égard, que les participes passés « masculins » et «invariables » (c'est-à-dire neutres) ont la même forme graphique... 
Ce qui tend d'ailleurs à corroborer ce point de vue est que, primo, il n'est pas toujours possible de féminiser un titre, et que, pour compliquer encore un peu plus les choses, certains «féminins » désignent l'épouse $d u$ tenant d'un titre et non pas la tenante du titre (présidente et ambassadrice, pour ne citer que ceux-la) ; secundo, le fait qu'il y ait une espèce de dévalorisation de la femme sous l'effet de la domination masculine, comme on a pu le souligner fort à propos, n'est pas une raison pour que nous entérinions et perpétuions les axiomes de la société patriarcale, en recourant à certains artifices (du genre «police-person ») qui ne font, en dernière analyse, que souligner les carences de notre système philosophico-social, et non pas de notre grammaire!

Aussi voudrais-je m'inscrire en faux contre l'affirmation que l'on avance quelquefois et selon laquelle certains noms n'ayant pas de féminin, les activités qui y correspondent dépasseraient nécessairement la compétence de la femme. II y a plus de trois ans déjà, on pouvait lire, dans la revue L'Express, "Madame X, premier ambassadeur féminin auprès du SaintSiège ». Cette tendance, qui se généralise de plus en plus, a l'énorme avantage de dépassionner un débat qui, je le répète, est le reflet d'une déformation de notre mentalité et non pas d'une faiblesse de notre langue.

Ainsi, c'est bien un souci de non-discrimination (par le biais de la nondifférenciation), et non pas 1'attitude inverse, qui devrait nous mener, en dehors d'ailleurs des considérations grammaticales et quelquefois euphoniques, à adopter une désignation unique pour les titres, en considérant que le « genre » du substantif correspondant n'a pas une connotation «sexiste », mais se réfère plutôt à la fonction visée qu'à la personne en cause, autre argument qui pousserait à opter pour ce « neutre » tellement commode qu'est ce « masculin grammatical » après tout... assez anodin.

DONALD GEENEN 